\title{
A NOVEL COMPUTATIONAL METHOD FOR COUPLED TWO POINT BOUNDARY VALUE PROBLEM
}

\author{
GANJI Narender ${ }^{1}$, KAMATAM Govardhan ${ }^{2}$ \\ ${ }^{1}$ Department of Humanities and Sciences (Mathematics), CVR College of Engineering, \\ Hyderabad-501510, Telangana State, India, e - mail: gnriimc@gmail.com \\ ${ }^{2}$ Department of Mathematics, GITAM University, Hyderabad-502329, Telangana State, India \\ e -mail: govardhan_kmtm@yahoo.co.in
}

\begin{abstract}
The paper deals with Finite difference Method of solving a boundary value problem involving a coupled pair of system of Ordinary Differential Equations. A novel iterative scheme is given for solving the Finite Difference Equations. Quasi-linearization used to convert a nonlinear problem into a series of linear problems. A problem from a flow of a nanofluid is presented as an example.
\end{abstract}

KEYWORDS: Quasi-linearization; Nanofluid; Dirichlet Condition; Finite Difference Method.

\section{Introduction}

Boundary value problems arising in Fluid Dynamics were treated by perturbation and asymptotic expansion methods before the advent of automatic computers. Complexity in the working of these methods increases with the nonlinearity of the underlying problems. HAM (homotopy analysis method) devised by Liao Shijan [1] was further improved later. It is a series expansion method that is not dependent on small or large physical parameters. Thus, it goes beyond some of the limitations of standard perturbation methods. The method needs a choice of basis functions to be used in the expansion. A class of popular methods is shooting methods. Among them, Newton method of finding missing initial conditions to satisfy the end conditions is a systematized one. In this paper nonlinear problems are converted into a series of linear problems by the well-known Quasi-linearization technique [2] to tackle a coupled system. Each liner problem is solved using finite differences and this system of finite difference equations is solved by a novel iterative technique. More literature regarding the sensor surface can be consulted in [3-5] applied to use the sensor surface model for the heat and mass transformation mechanisms.

We first consider linear coupled system and develop computational procedure using finite differences. Convergence of the iterative procedure used in solving linear system is rigorously established in section 2. A linear example is given in section 3. Non-linear problem is taken up in section 4. Conversion of non-linear problem into a series of linear problems by the Quasi-linearization technique is described. Each linear problem is solved using the method in section 2. We consider in section 5 a non-linear problem arising in nanofluids as an example. In discussion section 6, a technique is suggested for dealing with Neumann problem.

\section{Coupled Equations by Finite Difference Method}

$$
\begin{aligned}
& u^{\prime \prime}=f_{1}(x)+f_{2}(x) u+f_{3}(x) u^{\prime}+f_{4}(x) v+f_{5}(x) v^{\prime} \\
& v^{\prime \prime}=g_{1}(x)+g_{2}(x) v+g_{3}(x) v^{\prime}+g_{4}(x) u+g_{5}(x) u^{\prime}
\end{aligned}
$$


Here all the coefficients are continuous functions of $x$ over $[a, b]$.

Independent variable $x$ is in $[a, b]$ with boundary conditions

$$
u(a)=u_{a}, u(b)=u_{b}, v(a)=v_{a} \text {, and } v(b)=v_{b}
$$

with $h=\frac{b-a}{n}$, where $n$ is the number of intervals, derivatives are replaced by finite differences.

$$
\begin{gathered}
a=x_{0}, x_{1}, \ldots \ldots \ldots \ldots \ldots \ldots \ldots \ldots \ldots \ldots \ldots \ldots \ldots \ldots \\
u^{\prime}\left(x_{i}\right)=\frac{u_{i+1}-u_{i-1}}{2 h}, \quad u^{\prime \prime}\left(x_{i}\right)=\frac{u_{i+1}-2 u_{i}+u_{i-1}}{h^{2}} \\
v^{\prime}\left(x_{i}\right)=\frac{v_{i+1}-v_{i-1}}{2 h}, \quad v^{\prime \prime}\left(x_{i}\right)=\frac{v_{i+1}-2 v_{i}+v_{i-1}}{h^{2}}
\end{gathered}
$$

with $U=\left[u_{1}, u_{2}, \ldots \ldots . ., u_{n}\right]^{T}, V=\left[v_{1}, v_{2}, \ldots \ldots . ., v_{n}\right]^{T}$ are the column vectors.

The resulting system can be written as

$$
\begin{aligned}
& A_{1} U+A_{2} V=d \\
& A_{3} U+A_{4} V=e
\end{aligned}
$$

$A_{1}, A_{2}, A_{3}$ and $A_{4}$ are tridiagonal matrices.

$A_{1}=\left[a_{i, j}^{1}\right]$ where

$$
\begin{aligned}
a_{i, i}^{1} & =-\frac{2}{h^{2}}-f_{2}\left(x_{i}\right), i=1,2,3, \ldots \ldots \ldots, n-1 \\
a_{i, i+1}^{1} & =\frac{1}{h^{2}}-\frac{1}{2 h} f_{3}\left(x_{i}\right), i=1,2,3, \ldots \ldots . ., n-2 \\
a_{i, i-1}^{1} & =\frac{1}{h^{2}}+\frac{1}{2 h} f_{3}\left(x_{i}\right), i=1,2,3, \ldots \ldots . ., n-1
\end{aligned}
$$

$a_{n-1, n}^{1}, a_{0,1}^{1}$ go to right hand side after incorporating the boundary conditions.

$A_{2}=\left[a_{i, j}^{2}\right]$ where

$$
\begin{gathered}
a_{i, i}^{2}=f_{4}\left(x_{i}\right), i=1,2,3, \ldots \ldots \ldots \ldots, n-1 \\
a_{i, i+1}^{2}=-\frac{1}{2 h} f_{5}\left(x_{i}\right), i=1,2,3, \ldots \ldots \ldots \ldots ., n-2 \\
a_{i, i-1}^{2}=\frac{1}{2 h} f_{5}\left(x_{i}\right), i=0,1,2,3, \ldots \ldots . ., n-1
\end{gathered}
$$

$a_{n-1, n}^{2}, a_{0,1}^{2}$ go to right hand side after incorporating the boundary conditions with similar expressions for $A_{3}$ and $A_{4}$.

It may be noted that $\frac{1}{h^{2}}$ occurs in elements of $A_{1}$ and $A_{3}, \frac{1}{h}$ in $A_{2}$ and $A_{4}$. A measure of rate of convergence $-2 \ln (h)$ where spectral radius involves $\frac{1}{h^{2}}$.

Instead of solving these linear systems by direct matrix method we follow the following iterative procedure. 


\section{Algorithm:}

Step 1: Assuming initial approximation for the vector $V$, we solve the tri diagonal system $A_{1} U=d-A_{2} V$ for $U$. Any real $(n-1)$ vector ensures convergence as will be discussed later. We choose straight line passing through $\left(a, v_{a}\right)$ and $\left(b, v_{b}\right)$.

Step 2: Using this for $U$, we solve $A_{3} V=e-A_{4} U$.

Step 3: Go back to step 1 with this new $V$ if sufficient accuracy is not achieved.

To discuss the convergence of this iterative procedure, the method can be mathematically presented as described below.

$$
\begin{aligned}
& U=A_{1}^{-1}\left(d-A_{2} V\right) \\
& V=A_{3}^{-1}\left(e-A_{4} U\right)=A_{3}^{-1}\left(e-A_{4} A_{1}^{-1}\left(d-A_{2} V\right)\right)
\end{aligned}
$$

Thus

$$
V=A_{3}^{-1} A_{4} A_{1}^{-1} A_{2} V+A_{3}^{-1} e-A_{3}^{-1} A_{4} A_{1}^{-1} d
$$

The relation can formally be written as

$$
V=B V+p
$$

where $B=A_{3}^{-1} A_{4} A_{1}^{-1} A_{2} \quad d=-A_{3}^{-1} A_{4} A_{1}^{-1} d$

Convergence of this method is dependent on the spectral radius of the matrix $B$. It may be noted that two matrices should be non-singular. They are so if they are diagonally dominant. It is sufficient if the coefficients of $u$ and $v$ are nonnegative in their respective equations (3).

It is well known that the spectral radius of any matrix is less than any matrix norm defined on the matrix [8]. Now elements of $A_{1}$ contain $\frac{1}{h^{2}}$ as a factor; those of $A_{2}, \frac{1}{h} ; A_{3}$ contains $\frac{1}{h^{2}}$ as a factor while $A_{4}$ contains $\frac{1}{h}$. Thus $B$ contain $h^{2}$ as a factor; hence convergence is very fast, as the spectral radius is $o\left(h^{2}\right)$.

An example of a linear problem is presented in section 3 with results, followed by the introduction of the well-known Quasilinearization technique.

\section{Linear example}

In the problem, equations (1) and (2),

$$
\begin{gathered}
f_{1}(x)=x^{2}, f_{2}(x)=x, f_{3}(x)=2, f_{4}(x)=e^{x}, f_{5}(x)=1 . \\
g_{1}(x)=1, g_{2}(x)=x^{2}, g_{3}(x)=\operatorname{Sin}(x), g_{4}(x)=x, g_{5}(x)=3 . \\
u(0)=1, u(1)=2, v(0)=0 \text { and } v(1)=3 .
\end{gathered}
$$

$n=20$ is enough for three places accuracy as seen from the results. 
Table 1: Numerical Results

\begin{tabular}{|c|c|c|c|c|c|c|c|}
\hline \multicolumn{2}{|c|}{$x$} & $\mathbf{0}$ & $\mathbf{0 . 2}$ & $\mathbf{0 . 4}$ & $\mathbf{0 . 6}$ & $\mathbf{0 . 8}$ & $\mathbf{1 . 0}$ \\
\hline \multirow{2}{*}{$n=100$} & $u(x)$ & 1.00000 & 0.85282 & 0.75380 & 0.77417 & 1.07093 & 2.00000 \\
& $v(x)$ & 0.00000 & 0.36987 & 0.72522 & 1.13884 & 1.77121 & 3.00000 \\
\hline \multirow{2}{*}{$n=50$} & $u(x)$ & 1.00000 & 0.85288 & 0.75390 & 0.77428 & 1.07101 & 2.00000 \\
& $v(x)$ & 0.00000 & 0.36982 & 0.72515 & 1.13877 & 1.77116 & 3.00000 \\
\hline \multirow{2}{*}{$n=20$} & $u(x)$ & 1.00000 & 0.85277 & 0.75356 & 0.77361 & 1.07017 & 2.00000 \\
& $v(x)$ & 0.00000 & 0.36927 & 0.72408 & 1.13725 & 1.76958 & 3.00000 \\
\hline
\end{tabular}

The problem took 6 iterations to achieve a relative accuracy of $10^{-4}$.

\section{$4 \quad$ Nonlinear Problem}

We now consider the nonlinear system and

$$
\begin{aligned}
& u^{\prime \prime}=F\left(x, U, U^{\prime}, V, V^{\prime}\right) \\
& v^{\prime \prime}=F\left(x, V, V^{\prime}, U, U^{\prime}\right)
\end{aligned}
$$

with associated boundary conditions.

Let $U, V$ be initial approximations for $u$ and $v$ satisfying the given boundary conditions.

We linearize the problem by well-known Quasi-linearization Technique [2] as:

$$
u^{\prime \prime}=F\left(x, U, U^{\prime}, V, V^{\prime}\right)+(u-U) \frac{\partial F}{\partial u}+\left(u^{\prime}-U^{\prime}\right) \frac{\partial F}{\partial u^{\prime}}+(v-V) \frac{\partial F}{\partial v}+\left(v^{\prime}-V^{\prime}\right) \frac{\partial F}{\partial v^{\prime}}
$$

Here $U$ and $V$ are used in the partial derivatives on the right-hand side, and

$$
v^{\prime \prime}=F\left(x, V, V^{\prime}, U, U^{\prime}\right)+(v-V) \frac{\partial F}{\partial v}+\left(v^{\prime}-V^{\prime}\right) \frac{\partial F}{\partial v^{\prime}}+(u-U) \frac{\partial F}{\partial u}+\left(u^{\prime}-U^{\prime}\right) \frac{\partial F}{\partial u^{\prime}}
$$

This linear system is in $u$ and $v$ solved by the method described in section 2 .

If $|u-U|<\varepsilon$ and $|v-V|<\varepsilon$ where $\varepsilon$ is preassigned small number depending on the accuracy required, the resulting solution if final. If not with this small $u$ and $v$ as $U$ and $V$ go back to solve equations (7a) and (7b).

As an example of application of this method we consider a problem from Nanofluid.

\section{$5 \quad$ An example from Fluid Dynamics}

A mathematical model for analysing the convective Nanofluid over a linear stretching surface in the presence of nanoparticles and viscous dissipations is presented as an example. The governing partial differential equations (PDEs) are converted into a system of nonlinear ordinary differential equations (ODEs) using similarity transformations $[6,7]$. 


\section{Basic Equations:}

$$
\begin{gathered}
f^{\prime \prime \prime}+f f^{\prime \prime}-\left(f^{\prime}\right)^{2}=0, \\
\operatorname{Pr}^{-1} \theta^{\prime \prime}+f \theta^{\prime}+N b \theta^{\prime} \phi^{\prime}+N t\left(\theta^{\prime}\right)^{2}+E c\left(f^{\prime \prime}\right)^{2}=0, \\
\beta^{\prime \prime}+\operatorname{Le} f \beta^{\prime}+\frac{N t}{N b} \theta^{\prime \prime}=0 .
\end{gathered}
$$

Subject to the following boundary conditions:

$$
\begin{array}{lr}
f(\eta)=0, f^{\prime}(\eta)=1, \theta(\eta)=1, \beta(\eta)=1, & \text { at } \eta=0 \\
f^{\prime}(\eta) \rightarrow \infty, \theta(\eta) \rightarrow 0, \beta(\eta) \rightarrow 0, & \text { as } \eta=\infty
\end{array}
$$

Here the physics of the problem is described below:

$f \rightarrow$ velocity of the fluid, $\theta(\eta) \rightarrow$ Temperature of the fluid, $\beta(\eta) \rightarrow$ Nano particle concentration of the fluid.

Eq. (8) with the boundary conditions (11) and (12) has the analytical solution and is given by $f(\eta)=1-e^{-\eta}[7]$.

Quasi-linearization can be used for linearizing the nonlinear second order coupled ordinary differential equations

$$
\begin{gathered}
\theta^{\prime \prime}(\eta)=F\left(\theta, \theta^{\prime}, \beta, \beta^{\prime}\right)=-\operatorname{Pr}\left(f \theta^{\prime}+N b \theta^{\prime} \beta^{\prime}+N t\left(\theta^{\prime}\right)^{2}+E c\left(f^{\prime \prime}\right)^{2}\right) \\
\beta^{\prime \prime}(\eta)=G\left(\beta, \beta^{\prime}, \theta, \theta^{\prime}\right)=-L e f \beta^{\prime}+\frac{N t}{N b} \operatorname{Pr}\left(f \theta^{\prime}+N b \theta^{\prime} \beta^{\prime}+N t\left(\theta^{\prime}\right)^{2}+E c\left(f^{\prime \prime}\right)^{2}\right)
\end{gathered}
$$

The functions $\mathrm{F}$ and $\mathrm{G}$ can be expanded in a Taylor Series around a given $\theta_{0}, \beta_{0}$ and their derivatives $\theta_{0}^{\prime}$ and $\beta_{0}^{\prime}$

Thus

$$
\begin{aligned}
\theta^{\prime \prime}= & F\left(\theta_{0}, \theta_{0}^{\prime}, \beta_{0}, \beta_{0}^{\prime}\right)+\left(\theta-\theta_{0}\right)\left(\frac{\partial F}{\partial \theta}\right)_{0}+\left(\theta^{\prime}-\theta_{0}^{\prime}\right)\left(\frac{\partial F}{\partial \theta^{\prime}}\right)_{0}+\left(\beta-\beta_{0}\right)\left(\frac{\partial F}{\partial \beta}\right)_{0} \\
& +\left(\beta^{\prime}-\beta_{0}^{\prime}\right)\left(\frac{\partial F}{\partial \beta^{\prime}}\right)_{0}
\end{aligned}
$$

with similar expression for $\beta "$.

$$
\begin{aligned}
& \theta^{\prime \prime}=F_{0}+\theta^{\prime} F_{1}+\beta^{\prime} F_{2} \\
& \beta^{\prime \prime}=G_{0}+\beta^{\prime} G_{1}+\theta^{\prime} G_{2}
\end{aligned}
$$

where

$$
\begin{gathered}
F_{0}=-\operatorname{Pr}\left(f \theta_{0}^{\prime}+N b \theta_{0}^{\prime} \beta_{0}^{\prime}+N t\left(\theta_{0}^{\prime}\right)^{2}+E c\left(f^{\prime \prime}\right)^{2}\right)+\operatorname{Pr}\left(f+N b \beta_{0}^{\prime}+2 N t \theta_{0}^{\prime}\right) \theta_{0}^{\prime} \\
+\operatorname{Pr}\left(N b \theta_{0}^{\prime}\right) \beta_{0}^{\prime} \\
F_{1}=-\operatorname{Pr}\left(f+N b \beta_{0}^{\prime}+2 N t \theta_{0}^{\prime}\right) \\
F_{2}=-\operatorname{Pr}\left(N t \theta_{0}^{\prime}\right)
\end{gathered}
$$




$$
\begin{gathered}
G_{0}=- \text { Le } f \beta_{0}^{\prime}+\frac{N t}{N b} \operatorname{Pr}\left(f \theta_{0}^{\prime}+N b \theta_{0}^{\prime} \beta_{0}^{\prime}+N t\left(\theta_{0}^{\prime}\right)^{2}+E c\left(f^{\prime \prime}\right)^{2}\right)+L e f \\
+\frac{N t}{N b} \operatorname{Pr}\left(N b \theta_{0}^{\prime}\right) \beta_{0}^{\prime}+\frac{N t}{N b} \operatorname{Pr}\left(f+N b \beta_{0}^{\prime}+2 N t \theta_{0}^{\prime}\right) \theta_{0}^{\prime} \\
G_{1}=L e f+\frac{N t}{N b} \operatorname{Pr}\left(N b \theta_{0}^{\prime}\right) \text { and } \\
G_{2}=\frac{N t}{N b} \operatorname{Pr}\left(f+N b \beta_{0}^{\prime}+2 N t \theta_{0}^{\prime}\right) .
\end{gathered}
$$

Finite difference form of these differential equations can be written as

$$
\left.\begin{array}{l}
A_{1} U+A_{2} V=d, \\
A_{3} V+A_{4} U=e
\end{array}\right\}
$$

as presented in section 2 .

Iterative procedure of section 2 is implemented with the following observations being noted. The results compare excellently with those of Khan [7] and Narender [6] for the case $\operatorname{Pr}=1, N b=0.1, N t=0.1, L e=10, \eta_{\infty}=2$ (based on the reference [3]) and $h=0.01$.

Initial approximation for the Quasi Linear technique is chosen as the straight line passing through $(0,1)$ and $(2,0)$ for both $\theta$ and $\beta$.

To achieve a relative accuracy of $10^{-4}$, it took three outer iteration.

Initial approximation for each Q-L iteration is taken as a starting approximation for the matrix iteration. Three iteration are needed for the first Quasi-linearization iteration, two for the second iteration and one for the final Q-L iteration.

Possibly it needs more iterations for other values of physical parameters.

Table 2: Comparison of the computed results of Nusselt number and Sherwood number when $\operatorname{Pr}=L e=10, E c=0$.

\begin{tabular}{|c|c|c|c|c|c|c|c|}
\hline & & \multicolumn{3}{|c|}{$-\theta^{\prime}(0)$} & \multicolumn{3}{c|}{$-\beta^{\prime}(0)$} \\
\cline { 3 - 9 }$N t$ & $N b$ & $\begin{array}{c}\text { G. } \\
\text { Narender } \\
\text { [6] }\end{array}$ & $\begin{array}{c}\text { W.A.Khan, } \\
\text { I.Pop [7] }\end{array}$ & $\begin{array}{c}\text { Present } \\
\text { Result }\end{array}$ & $\begin{array}{c}\text { G. } \\
\text { Narender } \\
{[6]}\end{array}$ & $\begin{array}{c}\text { W.A.Khan, } \\
\text { I.Pop } \\
{[3]}\end{array}$ & $\begin{array}{c}\text { Present } \\
\text { Result }\end{array}$ \\
\hline 0.1 & 0.1 & 0.9523311 & 0.9524 & 0.9527659 & 2.129654 & 2.1294 & 2.1350480 \\
\hline 0.2 & 0.1 & 0.6930403 & 0.6932 & 0.6925833 & 2.275001 & 2.2740 & 2.2822810 \\
\hline 0.3 & 0.1 & 0.5197940 & 0.5201 & 0.5189359 & 2.531357 & 2.5286 & 2.5410710 \\
\hline 0.4 & 0.1 & 0.4020260 & 0.4026 & 0.4010332 & 2.801879 & 2.7952 & 2.8137410 \\
\hline 0.5 & 0.1 & 0.3200067 & 0.3211 & 0.3190041 & 3.050751 & 3.0351 & 3.0643050 \\
\hline 0.1 & 0.2 & 0.5055407 & 0.5056 & 0.5032206 & 2.382044 & 2.3819 & 2.3896270 \\
\hline 0.1 & 0.3 & 0.2521248 & 0.2522 & 0.2486408 & 2.410168 & 2.4100 & 2.4177900 \\
\hline 0.1 & 0.4 & 0.1193849 & 0.1194 & 0.1159728 & 2.399787 & 2.3997 & 2.4070310 \\
\hline 0.1 & 0.5 & 0.0542402 & 0.0543 & 0.0515175 & 2.383702 & 2.3836 & 2.3905780 \\
\hline
\end{tabular}

\section{Discussion with suggestion for solving Neumann problem}


System of linear equations involving a tri-diagonal matrix is known to be elegantly solved by Thomas algorithm. The method of solving a coupled system as suggested in this paper involves such tri diagonal matrices. With a Neumann condition, the resulting matrix, while using symmetric differences is not diagonally dominant. Method described in this paper does not work as such.

If one of the four boundary conditions for the two variables is a Neumann condition, we assume two values for the underlying variable in such a way the Neumann condition takes opposite signs. Using the Bisection method, we find the Dirichlet condition to describe degree of accuracy to solve the problem.

\section{CONCLUSION}

From the present study that has been numerically analyzed the following worthy points can be concluded:

- In the linear problem, $n=20$ is enough for three places accuracy as seen from the results.

- Initial approximation for each Q-L iteration is taken as a starting approximation for the matrix iteration.

- Three iteration are needed for the first Quasi-linearization iteration, two for the second iteration and one for the final Q-L iteration.

- Initial approximation for the Quasi Linear technique is chosen as the straight line.

\section{ACKNOWLEDGEMENT}

Help by Prf. S R Koneru, Retd. Professor, Department of Mathematics, IIT Mumbai in the preparation of this paper is gratefully acknowledged. We thank the referees for suggestions which enabled the paper highly presentable.

\section{REFERENCES}

[1] Liao, S. J. "The proposed homotopy analysis technique for the solution of nonlinear problems", PhD thesis, Shanghai Jiao Tong University, 1992.

[2] Bellman, R., Kalaba, R. "Quasilinearization and Boundary Value Problems", American Elsevier Publishing Company, New York, 1965.

[3] Kapilan N, Manjunath Gowda M., Manjunath H. N. "Computational Fluid Dynamics Analysis of an Evaporative Cooling System", Strojnícky Časopis - Journal of Mechanical Engineering 66 (2), pp. 117 - 124, 2016. DOI: 10.1515/scjme-2016-0026

[4] Gebauber, M., Brzobohaty, T., Blejchar, T., Karasek, T. "Numerical Simulation of Heat Treatment Process of Aluminium Profiles", Strojnícky časopis - Journal of Mechanical Engineering 69 (3), pp. 43 - 50, 2019. DOI: 10.2478/scjme-2019-0028

[5] P. Šlesar, R. Jančo. "Press-fit evaluation and study of displacement in temperature changes“, Strojnícky časopis - Journal of Mechanical Engineering 68 (1), pp. 103 108, 2018. DOI: $10.2478 /$ scjme-2018-0011

[6] Narender, G. "Computational Techniques on Heat and Mass Transfer in MHD Nanofluid flow over a stretching sheet", Ph.D. thesis submitted, Jawaharlal Nehru Technological University Hyderabad, HYDERABAD, Telangana State, India, 2020. 
[7] Khan, W.A., I. Pop. "Boundary-layer flow of a nanofluid past a stretching sheet", International Journal of Heat Mass Transfer 53, pp. 2477 - 2483, 2010.

[8] Varga, R. S. "Matrix Iterative Analysis", Prentice-Hall, Englewood Cliffs, N.J., 1962. 